\section{(2) OPEN ACCESS}

\title{
Sodium-glucose co-transporter 2 inhibitor therapy: mechanisms of action in heart failure
}

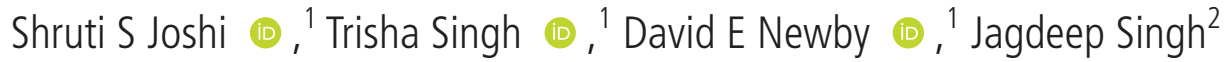

'BHF Centre for Cardiovascular Science, The University of Edinburgh, Edinburgh, UK 'Department of Cardiology, NHS Lothian, Edinburgh, UK

\section{Correspondence to}

Dr Shruti S Joshi, BHF Centre for Cardiovascular Science, The University of Edinburgh, Edinburgh, UK; sjoshi@ed.ac.uk

Received 19 August 2020 Revised 8 December 2020 Accepted 14 December 2020 Published Online First 26 February 2021
Check for updates

(C) Author(s) (or their employer(s)) 2021. Re-use permitted under CC BY. Published by BMJ.

To cite: Joshi SS, Singh T Newby DE, et al. Heart 2021;107:1032-1038.

\section{ABSTRACT}

Patients with type 2 diabetes mellitus are at a higher risk of developing heart failure compared with the healthy population. In recent landmark clinical trials, sodium-glucose co-transporter 2 (SGLT2) inhibitor therapies improve blood glucose control and also reduce cardiovascular events and heart failure hospitalisations in patients with type 2 diabetes. Intriguingly, such clinical benefits have also been seen in patients with heart failure in the absence of type 2 diabetes although the underlying mechanisms are not clearly understood. Potential pathways include improved glycaemic control, diuresis, weight reduction and reduction in blood pressure, but none fully explain the observed improvements in clinical outcomes. More recently, novel mechanisms have been proposed to explain these benefits that include improved cardiomyocyte calcium handling, enhanced myocardial energetics, induced autophagy and reduced epicardial fat. We provide an up-to-date review of cardiac-specific SGLT2 inhibitor-mediated mechanisms and highlight studies currently underway investigating some of the proposed mechanisms of action in cardiovascular health and disease.

\section{INTRODUCTION}

Heart failure is a global pandemic and the current worldwide prevalence of heart failure is estimated at 64.34 million cases accounting for 9.91 million years lost due to disability. ${ }^{1}$ The prevalence of heart failure increases with age and is associated with other comorbidities like type 2 diabetes mellitus, hypertension and obesity. People with type 2 diabetes are 2.5 times more likely to develop cardiac failure than people without diabetes. ${ }^{2}$ Concerns regarding cardiovascular adverse events associated with type 2 diabetes have conventionally focused around atherosclerotic events, but with major improvements in the treatment of myocardial infarction, stroke and limb ischaemia over the past few decades, heart failure is increasingly being recognised as one of its earliest and most serious complications. $^{3}$

The problems of heart failure associated with diabetes are compounded by the fact that several therapeutic glucose-lowering agents are either ineffective or harmful. Thiazolidinediones, such as pioglitazone, activate peroxisome proliferatoractivated receptor gamma (PPAR gamma) to improve insulin sensitivity. However, they also cause increased sodium and fluid retention, thereby leading to a $42 \%$ increased risk of incident heart failure. ${ }^{4}$ In contrast, dipeptidyl peptidase 4 inhibitors appear to have a neutral effect on major adverse cardiovascular events although one agent of this class, saxagliptin, has been associated with a $27 \%$ increase in hospitalisation for heart failure and a $22 \%$ increase in cardiovascular mortality. ${ }^{5}$ A meta-analysis of more than 24000 individuals from four large clinical trials reported that the use of insulin for type 2 diabetes was associated with a $27 \%$ increase in all-cause mortality and $23 \%$ increase in hospitalisation for heart failure. ${ }^{6}$ Looking at 100000 individuals in the 'real-world' setting of an administrative database, such treatment was associated with an odds ratio of 2.02 for all-cause mortality and 1.42 for heart failure hospitalisation. ${ }^{6}$ Therefore, there is an unmet need for safer and more effective glucose-lowering therapies for patients with type 2 diabetes and heart failure.

\section{Sodium-glucose co-transporter 2 inhibitor therapy}

Sodium-glucose co-transporter 2 (SGLT2) inhibitors are a new class of glucose-lowering drugs. They work by blocking the low-affinity, high-capacity SGLT2 protein located in the proximal convoluted tubule of the nephron. The SGLT2 protein is responsible for the resorption of approximately $90 \%$ of filtered glucose while the remainder is reabsorbed by SGLT1 proteins found on the distal part of the proximal convoluted tubule. SGLT2 inhibition results in glycosuria (and natriuresis as the protein is a co-transporter), thereby lowering plasma glucose concentrations. ${ }^{78}$ This mechanism is unique compared with all other glucose-lowering agents as it does not interfere with endogenous insulin or incretin pathways.

In recent cardiovascular outcome trials, SGLT2 inhibitors are associated with 30\%-35\% lower risk of hospitalisation for heart failure. ${ }^{9-12}$ Other glucose-lowering agents appear to be more potent than SGLT2 inhibitors, but fail to reduce cardiovascular risk, particularly with regard to heart failure outcomes. Moreover, although the glucose-lowering efficacy of SGLT2-inhibitor therapy declines at lower estimated glomerular filtration rates, its cardiovascular benefits are remarkably preserved, even in patients with renal impairment. This implies differing mechanisms of action in glycaemic control and cardiovascular risk reduction. ${ }^{13}$ These potential cardioprotective mechanisms remain incompletely understood and we aim to describe some of the key theories (table 1) in this review as this could pave the way for targeting future novel therapies for both heart failure and type 2 diabetes.

\section{SGLT2 inhibitor therapy and cardiovascular outcomes}

In response to concerns about the increased cardiovascular risk associated with some treatments for 
Table 1 Potential mechanisms of cardiovascular benefits associated with SGLT2-inhibitor therapy

\begin{tabular}{ll}
\hline Conventional mechanisms & Novel mechanisms \\
\hline Diuresis and reduction in blood pressure & Improved myocardial energetics \\
Improved glycaemic control & Improved myocardial ionic homeostasis \\
$\begin{array}{l}\text { Weight loss } \\
\begin{array}{l}\text { Increase in red blood cell mass and } \\
\text { haematocrit }\end{array}\end{array}$ & Autophagy \\
\hline
\end{tabular}

diabetes, regulators in the USA and Europe mandated cardiovascular safety trials of all new hypoglycaemic drugs in 2008. In recent cardiovascular outcome trials, the glucagon-like peptide 1 receptor agonist (GLP-1 RA) and SGLT2 inhibitors have demonstrated reductions in cardiovascular events, reversing the trend of previous therapies. While the benefits with GLP1RAs are associated with reduced atherothrombotic events like myocardial infarction and stroke, ${ }^{14}$ SGLT2-inhibitor therapies have demonstrated improved outcomes in heart failure ${ }^{9} 1015$ (table 2). Interestingly, the reduction in heart failure hospitalisation was observed within months of randomisation, suggesting a different mechanism of effect from those of other glucoselowering drugs which usually takes years to manifest. ${ }^{9-12}$ The consistent signal of improved heart failure outcomes in the early trials led to the 'Dapagliflozin in Patients with Heart Failure and Reduced Ejection Fraction' (DAPA-HF trial) which was specifically designed to evaluate the efficacy of the SGLT2-inhibitor therapy in patients with heart failure and a reduced ejection fraction, regardless of the presence or absence of diabetes. This trial showed that dapagliflozin reduces the risk of worsening heart failure or death from cardiovascular causes by $26 \%$, regardless of diabetes status, suggesting that these benefits are independent of the drug's glucose-lowering effect. ${ }^{151}$ More recently, results from the 'Empagliflozin outcome trial in patients with chronic heart failure with reduced ejection fraction' (EMPEROR-Reduced) trial showed that empagliflozin also reduced the composite risk of cardiovascular death and heart failure hospitalisation in patients with known heart failure with reduced ejection fraction regardless of diabetes status. ${ }^{16}$ In both of these trials, the risk reduction seen in their primary outcomes was principally driven by a reduction in hospitalisations for heart failure. ${ }^{15} 16$ Meta-analysis of these two trials shows consistency of effect of both dapagliflozin and empagliflozin with reductions in all the individual endpoints of all-cause death, cardiovascular death, hospitalisation for heart failure or decline in renal function. ${ }^{17}$ Thus, these striking benefits appear to be a class effect of SGLT2inhibitor therapies. So, what is the mechanism of these marked benefits?

\section{Conventional potential mechanisms of benefit with SGLT2 inhibition}

The desirable cardiovascular effects seen with SGLT2 inhibitor treatment may be multifactorial, but key pathways involved are currently being explored. Some of the conventional theories are discussed in the following section.

\section{Diuretic and antihypertensive effects}

It has been suggested that SGLT2 inhibitors improve cardiovascular outcomes due to their diuretic and antihypertensive effects (figure 1). SGLT2 inhibitors cause osmotic diuresis due to glucosuria and natriuresis, although the degree of diuresis and its composition (ie, the amount of natriuresis compared with aquaresis) remains to be established. ${ }^{18}$ Indeed, in the context of co-administration of loop diuretic therapies, the contribution of osmotic diuresis towards improved heart failure outcomes remains unclear. Glycosuria in the context of SGLT2-inhibitor therapy administration occurs due to a twofold to threefold increase in the filtered and resorbed glucose in presence of hyperglycaemia. Therefore, glycosuria and osmotic diuresis associated with SGLT2 inhibition are dependent on blood glucose concentrations and hence this does not explain the similar benefits observed in normoglycaemic patients with heart failure in the absence of diabetes mellitus. ${ }^{15} 16$ Although SGLT2inhibitor therapy is associated with natriuresis and reduction in plasma volume, ${ }^{919}$ it is unclear if these benefits are sustained as there were no differences seen in serum $\mathrm{N}$-terminal pro B-type natriuretic peptide (NT-pro BNP) concentrations in patients with chronic stable heart failure despite improvements in heart failure status. ${ }^{20}$ Moreover, there was no change in the diuretic dose among most participants during follow-up in the DAPA-HF trial, and the mean diuretic dose was similar between dapagliflozin and placebo groups. ${ }^{15}$

Recent studies have highlighted important differences between the diuretic effect of SGLT2 inhibitors and loop diuretic therapies. Using mathematical modelling, a recent study showed that both dapagliflozin and bumetanide were associated with a reduction in sodium and interstitial fluid. However, dapagliflozin had little or no effect on plasma volume whereas bumetanide was associated with reductions in intravascular volume, which can pose challenges in the context of hypoperfusion. ${ }^{21}$ This predicted selectivity of SGLT2 inhibitors for interstitial fluid is supported by observations from 'the renal and cardiovascular effects of SGLT2 inhibition in combination with loop diuretics in patients with type 2 diabetes and chronic heart failure' (RECEDE-CHF) trial which showed an average increase in daily urine volume of $545 \mathrm{~mL}, 312 \mathrm{~mL}$ of which was electrolyte-free water clearance. $^{22}$ This may suggest a synergistic effect of SGLT2-inhibitor therapy with background diuretic treatment in heart failure. This, however, requires further validation.

The antihypertensive effect associated with SGLT2 inhibition was previously thought to be secondary to diuresis and natriuresis, but given that this effect is preserved even with declining glomerular filtration rates, it has been suggested to be more likely secondary to improved endothelial function, reduced arterial stiffness and changes in sympathetic nervous activity as evidenced by recent studies. ${ }^{23} 24$ A recent meta-analysis reported only a modest overall antihypertensive effect from SGLT2 inhibition: pooled estimate reduction of $2.46 / 1.46 \mathrm{~mm} \mathrm{Hg}$ in blood pressure. ${ }^{25}$ This degree of reduction in blood pressure, although salutary in the context of cardiovascular disease, is also unlikely to be responsible for the striking benefits in cardiovascular morbidity and mortality.

\section{Weight reduction and improved glycaemic control}

It has been postulated that weight reduction and improved glycaemic control underlie the cardioprotective effect seen with SGLT2-inhibitor therapy. However, there are key considerations worthy of discussion. Weight loss from SGLT2-inhibitor therapy occurs due to an increased glucagon:insulin ratio causing increased lipid mobilisation and is thought to be one of the mechanisms involved in reduction in heart failure mortality associated with SGLT2-inhibitor therapy. ${ }^{26} 27 \mathrm{Up}$ to $2.7 \mathrm{~kg}$ weight loss was observed in patients with type 2 diabetes with SGLT2-inhibitor therapy, and there are some studies suggesting weight loss in prediabetic patients. ${ }^{28}{ }^{29}$ However, there is currently no evidence to suggest that it can cause weight loss in patients with heart failure 

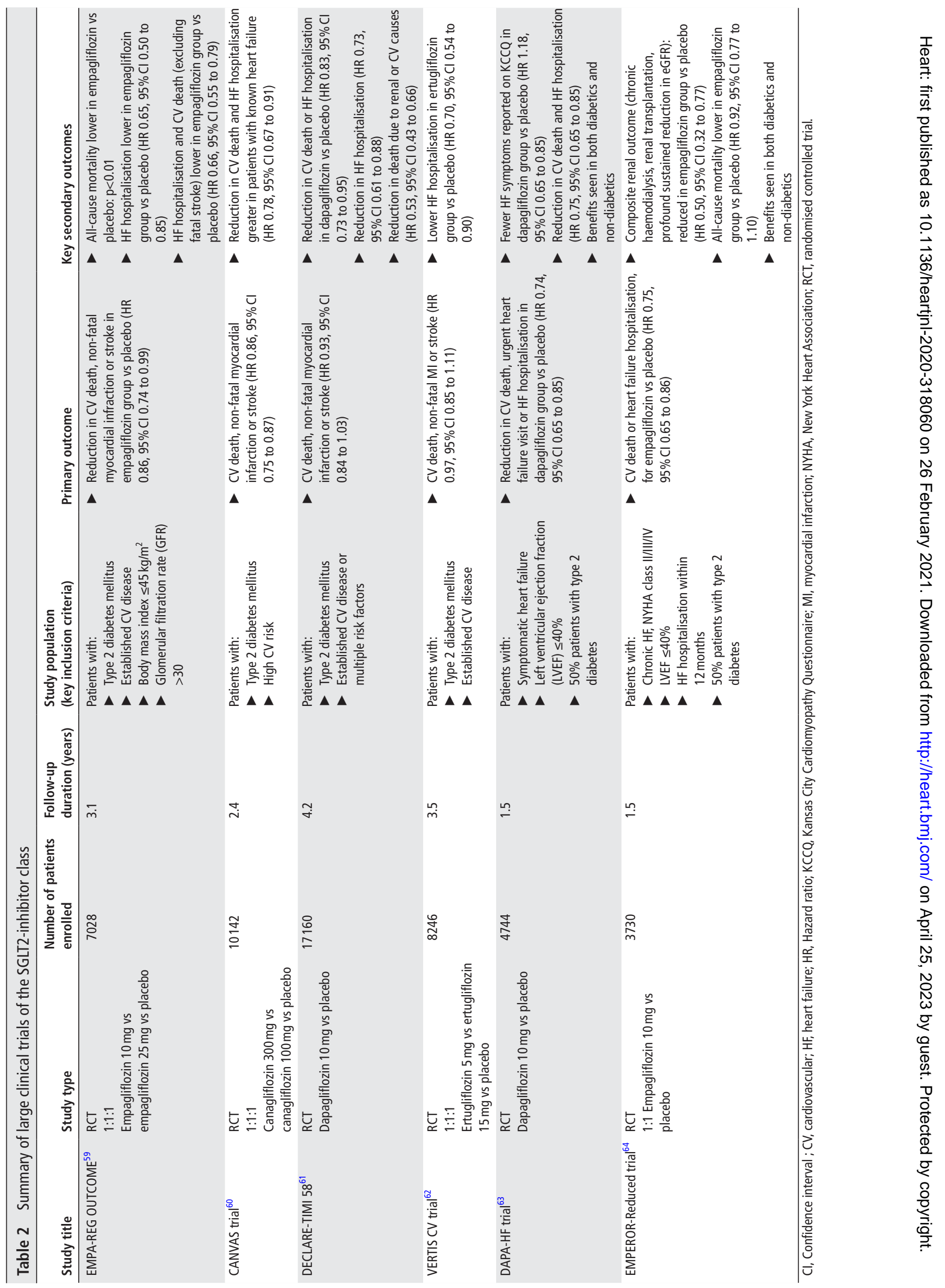


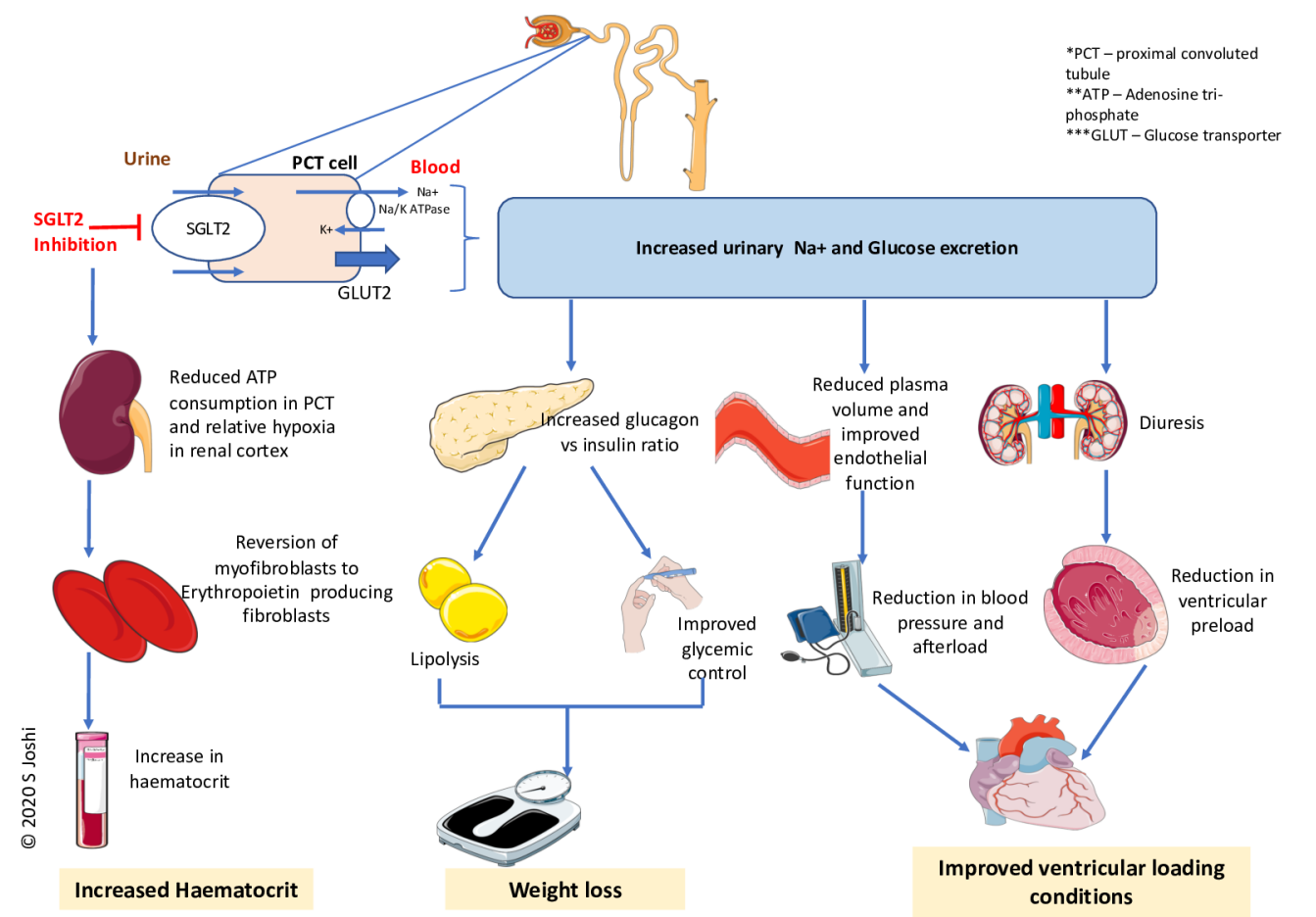

in the absence of diabetes which would be an argument against weight reduction being the primary mechanism of benefit with SGLT2-inhibitor therapy. Moreover, despite the high prevalence of obesity in heart failure, there is little definitive evidence regarding the impact of weight loss on cardiac function, quality of life and exercise tolerance in patients with heart failure. ${ }^{30}$ Therefore, weight loss alone cannot explain SGLT2 inhibitionrelated benefits in heart failure.

\section{Increase in haematocrit}

SGLT2-inhibitor therapy is associated with increases in renal erythropoietin production, red blood cell mass and haematocrit. $^{27}$ Such changes may contribute to improvements in cardiovascular outcomes although similar increases in haematocrit have been reported with darbepoetin alfa and no mortality benefit was observed in patients with left ventricular systolic dysfunction. ${ }^{31}$

Overall, many of these mechanisms and recognised factors have been associated with reductions in cardiovascular risk (figure 1). However, the modest improvements seen with SGLT2-inhibitor therapy in these domains do not provide a clear explanation for the striking benefits observed in heart failure events in the large clinical trials and key pathways involved still require further investigation.

\section{Novel potential mechanisms of benefit with SGLT2 inhibition Direct cardiac effects of SGLT2-inhibitor therapy}

Cardiac hypertrophy, fibrosis and inflammation lead to adverse cardiac remodelling in heart failure, and this is a key contributor to its severity. ${ }^{32}$ Some pre-clinical and clinical studies have demonstrated a role for SGLT2-inhibitor therapies in reversing adverse cardiac remodelling. ${ }^{33} 34$ Although this effect has been observed in patients with type 2 diabetes and left ventricular hypertrophy, it has not been seen in patients with heart failure. ${ }^{35} 36$ This is intriguing, particularly, since the majority of its cardiovascular benefits have been around heart failure outcomes. This raises the possibility that, in the context of heart failure, SGLT2 inhibition may have novel direct cardioprotective effects, beyond that of ventricular loading and remodelling (figure 2).

\section{Improved myocardial energetics}

Under physiological conditions, nearly $90 \%$ of cardiac energy is derived from mitochondrial oxidative metabolism and fuel is derived from free fatty acids, glucose and to a lesser extent from lactate, ketones and amino acids. ${ }^{37}$ In type 2 diabetes or heart failure, there is dysregulated fatty acid oxidation and impaired glucose uptake or oxidation causing myocardial dysfunction. In this setting of restricted fuel selection and low energetic reserve, ketone bodies are a 'super-fuel', producing ATP more efficiently than glucose or free fatty acids. SGLT2-inhibitor therapy increases hepatic synthesis and decreases the urinary excretion of ketones producing a mild and persistent state of hyperketonaemia. Under these conditions, beta-hydroxybutyrate (ketone body) is freely taken up by the heart and kidney and oxidised in preference to fatty acids and glucose. In mouse hearts, betahydroxybutyrate increases external cardiac work and reduces oxygen consumption, thereby improving cardiac efficiency. ${ }^{38}$ It has thus been hypothesised that the cardiovascular benefits of SGLT2-inhibitor therapy may be related to a shift in cardiac metabolism away from fatty acids and glucose oxidation towards more oxygen-efficient ketone bodies, thereby improving cardiac efficiency.

Improved ionic homeostasis in the myocardium

Calcium homeostasis in the myocardium is a finely balanced mechanism, essential for efficient excitation-contraction coupling. ${ }^{39}$ During systole, calcium is actively transported into the cardiomyocyte via L-type voltage-gated calcium channels where it binds to ryanodine receptors on the sarcoplasmic reticulum resulting in 'calcium-induced calcium release'. This, in turn, activates calcium-sensitive contractile proteins (troponin C, troponin NC) which leads to myocardial contraction. In the setting of type 2 diabetes and heart failure, there is upregulation 


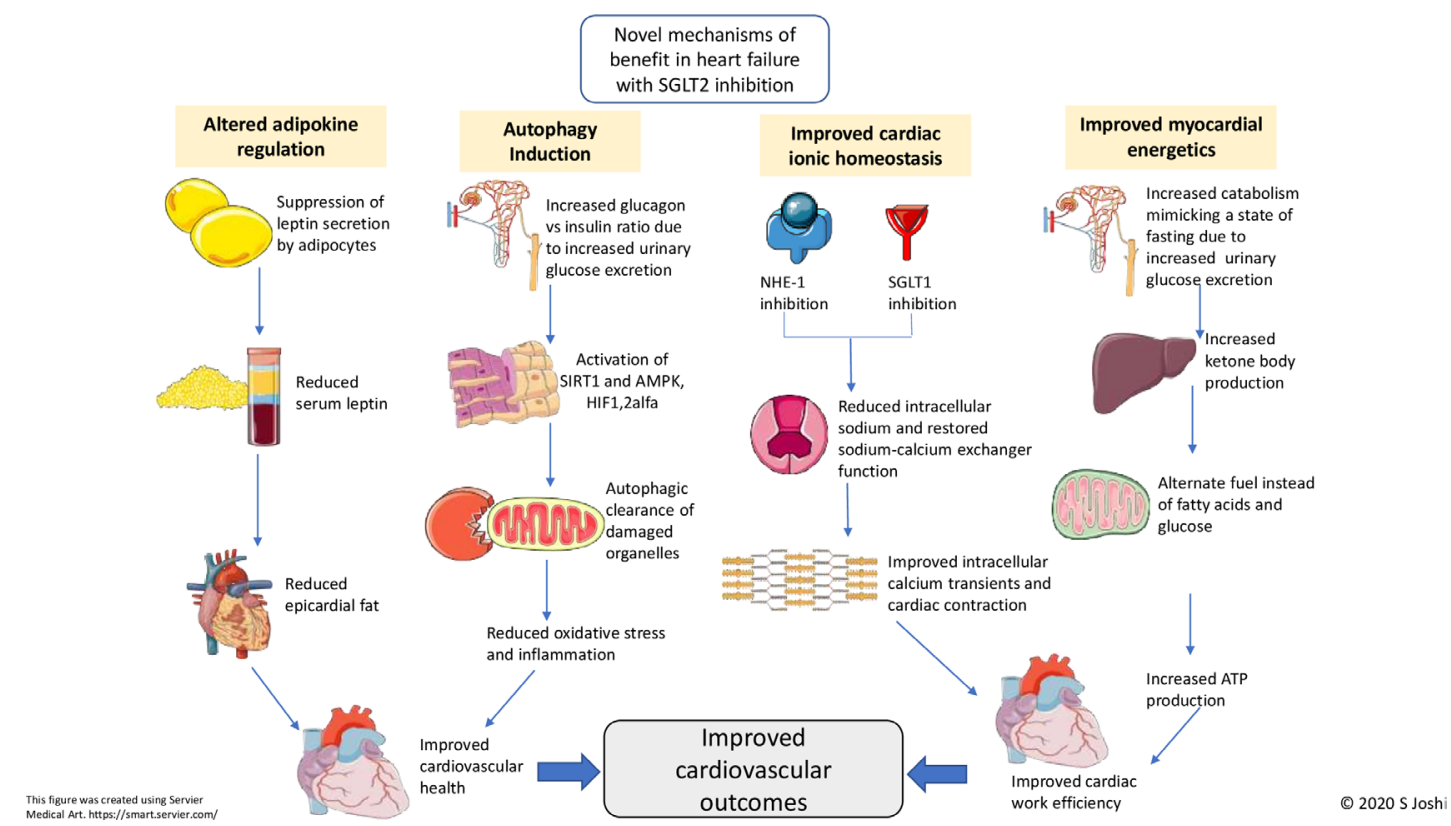

of both sodium-hydrogen exchanger 1 and SGLT1 resulting in markedly increased intra-cytosolic sodium content. ${ }^{40} 41$ This promotes calcium influx via the membrane bound sodiumcalcium exchanger transporters and calcium efflux from the mitochondria (into the cytosol) via mitochondrial sodiumcalcium exchanger transporters. The elevated baseline intracellular calcium content results in reduced calcium transients and smaller sarcoplasmic reticulum calcium stores in diabetic cardiomyocytes, thereby inhibiting contractile function. ${ }^{42}$

SGLT2-inhibitor therapy reduces cardiac cytosolic sodium content by inhibiting sodium-hydrogen exchanger 1 and SGLT1 transporters in diabetic rat and mice myocytes, thereby reversing calcium overload. ${ }^{42-47}$ Intriguingly, this effect on sodiumhydrogen exchanger 1 and SGLT1 is independent of diabetes status. ${ }^{48}$ These findings suggest that altered myocardial calcium handling is involved in the development of diabetic cardiomyopathy and heart failure, and that SGLT2-inhibitor therapy may improve the electrochemical characteristics in the failing myocardium which may contribute to its cardiovascular benefit. An ongoing clinical trial will assess the role of altered calcium handling in diabetic cardiomyopathy and heart failure and determine the effects of SGLT2-inhibitor therapy on cardiac calcium homeostasis (NCT04591639). The study uses a novel imaging approach called manganese-enhanced magnetic resonance imaging (MRI). Manganese acts as a calcium analogue and administration of the manganese-based contrast agent results in a marked shortening of the $\mathrm{T} 1$ relaxation times in myocardium with intact calcium handling. The rate of T1 shortening therefore acts as a measure of myocardial calcium handling, and this is the primary endpoint of the study.

\section{Autophagy}

Autophagy is the process by which cellular physiological equilibrium is maintained by removal of potentially dangerous constituents and recycling of cellular components as an adaptive response to metabolic stress including hypoxia and starvation. ${ }^{49}$ Experimental induction of autophagy has favourable effects in heart failure as it leads to efficient disposal of dysfunctional mitochondria, which are a major source of reactive oxygen species, promoting oxidative stress and inflammation. ${ }^{50}$ The pathways of autophagy induction involve activation of adenosine monophosphate-activated protein kinase (AMPK), sirtuin-1 (SIRT1) and hypoxia-inducible factors (HIF-1alfa and HIF-2alfa). ${ }^{51}$ It has been proposed that SGLT2 inhibitors may induce autophagy by simulating nutrient depletion by periods of increased catabolism due to constant glucosuria. Indeed, various SGLT2-inhibitor therapies have upregulated the expression of AMPK, SIRT1 and HIF-1 alfa. ${ }^{53}$ These actions might explain the phenomenon of autophagy and associated cardiovascular benefits with SGLT2-inhibitor therapy. ${ }^{54}$

\section{Altered adipokine regulation}

Leptin and adiponectin are cytokines produced exclusively by adipocytes. These 'adipokines' are essential in the regulation of food intake and energy homeostasis with leptin being implicated in various obesity-related cardiovascular diseases, whereas adiponectin is considered cardioprotective. ${ }^{55}$ Epicardial fat deposition due to altered adiponectin and leptin regulation is one of the theories implicated in the development of heart failure. ${ }^{56}$ Increased serum leptin concentrations are seen in patients with heart failure and they are associated with cardiac remodelling due to cardiac fibrosis and inflammation. ${ }^{57}$

SGLT2 inhibition reduces serum leptin and increases adiponectin concentrations, potentially offering some cardioprotection. ${ }^{58}$ However, these effects may well be reflective of changes secondary to the systemic effects of SGLT2-inhibitor therapy including weight loss and lipolysis.

\section{Future directions}

The exact pathways of cardiovascular benefit with SGLT2inhibitor therapy are yet to be established and newer mechanisms may emerge over time. It is plausible that SGLT2 inhibition interacts with or mediates other key pathways at a cellular level, facilitating cardiovascular benefits. Thus, establishing the exact mechanism of benefit is key to understanding the benefits of 
SGLT2 inhibition and may also open up new and unexplored pathways which could provide rich avenues of understanding for the pathophysiology and potential future novel treatments of heart failure.

There are several ongoing clinical studies investigating the mechanisms of cardiovascular benefits with SGLT2 inhibition such as the effects on cardiac remodelling (NCT03871621), lipolysis and modification of epicardial fat thickness and properties (NCT04219124, NCT04167761 and NCT02235298), myocardial calcium handling (NCT04591639) and endogenous ketone production (NCT03852901, NCT04219124).

These studies will provide significant insights into the key pathways involved in the cardioprotective effects associated with SGLT2-inhibitor therapy.

\section{CONCLUSIONS}

SGLT2-inhibitor therapies are a promising new class of drugs for treating type 2 diabetes and heart failure. The cardioprotective effect of SGLT2 inhibition has been demonstrated in models of diabetic cardiomyopathy, heart failure and ischaemic cardiomyopathy. The mechanism through which SGLT2-inhibitor therapy exerts its benefit in patients with heart failure remains incompletely understood. It is possible that a combination of systemic and direct effects of SGLT2 inhibition on the myocardium ultimately leads to the cardiovascular benefits. However, there is strong pre-clinical evidence to suggest that improved myocardial ionic homeostasis has a role to play with regards to the cardiovascular benefits seen with SGLT2-inhibitor therapy. Studies examining the mechanistic role of SGLT2 inhibitor therapy in cardiovascular health will be instrumental in shaping our understanding of heart failure and diabetic cardiomyopathy and may open avenues for the development of future drug therapies that target these pathways.

Correction notice This article has been corrected since it first published. The provenance and peer review statement has been included.

Contributors SSJ drafted the manuscript, which was critically reviewed by TS, DEN and JS. All authors approved the final submission.

Funding The authors are supported by British Heart Foundation (SJ, RE/18/5/3 and FS/CRTF/20/24087; TS RE/18/5/3; DEN, CH/09/002, RG 16/10/3, RE 18/5/3), Medical Research Council (TS, MR/T029153/1), Wellcome Trust (DEN, WT103782AIA) and an investigator initiated award from AstraZeneca (SJ, SER-19-20118).

Competing interests The authors have received an investigator initiated award from AstraZeneca to conduct the 'DAPA-MEMRI' study.

Patient consent for publication Not required.

Provenance and peer review Commissioned; externally peer reviewed.

Supplemental material This content has been supplied by the author(s). It has not been vetted by BMJ Publishing Group Limited (BMJ) and may not have been peer-reviewed. Any opinions or recommendations discussed are solely those of the author(s) and are not endorsed by BMJ. BMJ disclaims all liability and responsibility arising from any reliance placed on the content. Where the content includes any translated material, BMJ does not warrant the accuracy and reliability of the translations (including but not limited to local regulations, clinical guidelines, terminology, drug names and drug dosages), and is not responsible for any error and/or omissions arising from translation and adaptation or otherwise.

Open access This is an open access article distributed in accordance with the Creative Commons Attribution 4.0 Unported (CC BY 4.0) license, which permits others to copy, redistribute, remix, transform and build upon this work for any purpose, provided the original work is properly cited, a link to the licence is given, and indication of whether changes were made. See: https://creativecommons.org/ licenses/by/4.0/

\section{ORCID iDs}

Shruti S Joshi http://orcid.org/0000-0001-9874-6211

Trisha Singh http://orcid.org/0000-0002-4314-9935

David E Newby http://orcid.org/0000-0001-7971-4628

\section{REFERENCES}

1 Lippi G, Sanchis-Gomar F. Global epidemiology and future trends of heart failure. AME Med J 2020;5:15. doi:10.21037/amj.2020.03.03

2 Maack C, Lehrke M, Backs J, et al. Heart failure and diabetes: metabolic alterations and therapeutic interventions: a state-of-the-art review from the Translational Research Committee of the Heart Failure Association-European Society of Cardiology. Eur Heart J 2018:39:4243-54.

3 Packer M. Heart failure: the most important, preventable, and treatable cardiovascular complication of type 2 diabetes. Diabetes Care 2018;41:11-13.

4 Udell JA, Cavender MA, Bhatt DL, et al. Glucose-lowering drugs or strategies and cardiovascular outcomes in patients with or at risk for type 2 diabetes: a meta-analysis of randomised controlled trials. Lancet Diabetes Endocrinol 2015:3:356-66.

5 Scirica BM, Bhatt DL, Braunwald E, et al. Saxagliptin and cardiovascular outcomes in patients with type 2 diabetes mellitus. N Eng/ J Med 2013;369:1317-26.

6 Cosmi F, Shen L, Magnoli M, et al. Treatment with insulin is associated with worse outcome in patients with chronic heart failure and diabetes. Eur J Heart Fail 2018;20:888-95.

7 Hummel CS, Lu C, DDF L. Glucose transport by human renal Na+/D-glucose cotransporters SGLT1 and SGLT2. Am J Physiol Physiol 2011;300:C14-21.

8 Kalra S. Sodium glucose co-transporter-2 (SGLT2) inhibitors: a review of their basic and clinical pharmacology. Diabetes Ther 2014;5:355-66.

9 Zinman B, Wanner C, Lachin JM, et al. Empagliflozin, cardiovascular outcomes, and mortality in type 2 diabetes. N Engl J Med 2015;373:2117-28.

10 Neal B, Perkovic V, Mahaffey KW. Canagliflozin and cardiovascular and renal events in type 2 diabetes. N Engl J Med 2017;377:644-57.

11 Wiviott SD, Raz I, Bonaca MP. Dapagliflozin and cardiovascular outcomes in type 2 diabetes. N Eng/ J Med 2019;380:347-57.

12 Cannon CP, Pratley R, Dagogo-Jack S. Cardiovascular outcomes with ertugliflozin in type 2 diabetes. N Engl J Med 2020;383:1425-35.

13 Kosiborod M, Lam CSP, Kohsaka S, et al. Cardiovascular events associated with SGLT-2 inhibitors versus other glucose-lowering drugs: the CVD-REAL 2 study. J Am Coll Cardiol 2018;71:2628-39.

14 Kim GS, Park JH, Won JC. The role of glucagon-like peptide 1 receptor agonists and sodium-glucose cotransporter 2 inhibitors in reducing cardiovascular events in patients with type 2 diabetes. Endocrinol Metab 2019;34:106.

15 McMurray JJV, Solomon SD, Inzucchi SE. Dapagliflozin in patients with heart failure and reduced ejection fraction. N Eng/ J Med 2019;381:1995-2008.

16 Packer M, Anker SD, Butler J. Cardiovascular and renal outcomes with empagliflozin in heart failure. N Engl J Med 2020:NEJMoa2022190.

17 Zannad F, Ferreira JP, Pocock SJ, et al. SGLT2 inhibitors in patients with heart failure with reduced ejection fraction: a meta-analysis of the EMPEROR-Reduced and DAPAHF trials. Lancet 2020;396:819-29.

18 Sha S, Polidori D, Heise T, et al. Effect of the sodium glucose co-transporter 2 inhibitor canagliflozin on plasma volume in patients with type 2 diabetes mellitus. Diabetes Obes Metab 2014;16:1087-95.

19 Dekkers CCJ, Sjöström CD, Greasley PJ, et al. Effects of the sodium-glucose cotransporter-2 inhibitor dapagliflozin on estimated plasma volume in patients with type 2 diabetes. Diabetes Obes Metab 2019;21:2667-73.

20 Nassif ME, Windsor SL, Tang F, et al. Dapagliflozin effects on biomarkers, symptoms, and functional status in patients with heart failure with reduced ejection fraction: the DEFINE-HF trial. Circulation 2019;140:1463-76.

21 Hallow KM, Helmlinger G, Greasley PJ, et al. Why do SGLT2 inhibitors reduce heart failure hospitalization? A differential volume regulation hypothesis. Diabetes Obes Metab 2018;20:479-87.

22 Mordi NA, Mordi IR, Singh JS, et al. Renal and cardiovascular effects of SGLT2 inhibition in combination with loop diuretics in patients with type 2 diabetes and chronic heart failure: the RECEDE-CHF trial. Circulation 2020;142:1713-24. doi:10.1161/CIRCULATIONAHA.120.048739

23 Cherney DZ, Perkins BA, Soleymanlou N, et al. The effect of empagliflozin on arteria stiffness and heart rate variability in subjects with uncomplicated type 1 diabetes mellitus. Cardiovasc Diabetol 2014;13:28.

24 Pfeifer M, Townsend RR, Davies MJ, et al. Effects of canagliflozin, a sodium glucose co-transporter 2 inhibitor, on blood pressure and markers of arterial stiffness in patients with type 2 diabetes mellitus: a post hoc analysis. Cardiovasc Diabetol 2017;16:29.

25 Mazidi M, Rezaie P, Gao H-K, et al. Effect of sodium-glucose cotransport-2 inhibitors on blood pressure in people with type 2 diabetes mellitus: a systematic review and meta-analysis of 43 randomized control trials with 22528 patients. J Am Heart Assoc 2017;6. doi:10.1161/JAHA.116.004007

26 Nakayama H, Ohtsuka Y, Kawahara M. Changes in body composition during SGLT2 inhibitor treatment and their relevance to the improvement of insulin sensitivity. Diabetes Res Clin Pract 2016;120:S50-1.

27 lemitsu K, lizuka T, Takihata M, et al. Factors influencing changes in hemoglobin A1c and body weight during treatment of type 2 diabetes with ipragliflozin: interim analysis of the ASSIGN-K study. J Clin Med Res 2016;8:373-8. 
28 Pinto L, Rados D, Remonti L. Efficacy of SGLT2 inhibitors in glycemic control, weight loss and blood pressure reduction: a systematic review and meta-analysis. Diabetol Metab Syndr 2015;7:A58.

29 Ramírez-Rodríguez AM, González-Ortiz M, Martínez-Abundis E. Effect of dapagliflozin on insulin secretion and insulin sensitivity in patients with prediabetes. Exp Clin Endocrinol Diabetes 2020;128:506-511. doi:10.1055/a-0664-7583

30 McDowell K, Petrie MC, Raihan NA, et al. Effects of intentional weight loss in patients with obesity and heart failure: a systematic review. Obes Rev 2018;19:1189-204.

31 Swedberg K, Young JB, Anand IS, et al. Treatment of anemia with darbepoetin alfa in systolic heart failure. N Engl J Med 2013;368:1210-9.

32 Heusch G, Libby P, Gersh B, et al. Cardiovascular remodelling in coronary artery disease and heart failure. Lancet 2014;383:1933-43.

33 Shi L, Zhu D, Wang S, et al. Dapagliflozin attenuates cardiac remodeling in mice model of cardiac pressure overload. Am J Hypertens 2019;32:452-9.

34 Lee H-C, Shiou Y-L, Jhuo S-J, et al. The sodium-glucose co-transporter 2 inhibitor empagliflozin attenuates cardiac fibrosis and improves ventricular hemodynamics in hypertensive heart failure rats. Cardiovasc Diabetol 2019;18:45.

35 Verma S, Mazer CD, Yan AT, et al. Effect of empagliflozin on left ventricular mass in patients with type 2 diabetes mellitus and coronary artery disease: the EMPA-HEART CardioLink-6 randomized clinical trial. Circulation 2019;140:1693-702.

36 Singh JSS, Mordi IR, Vickneson K, et al. Dapagliflozin versus placebo on left ventricular remodeling in patients with diabetes and heart failure: the REFORM trial. Diabetes Care 2020;43:dc192187

37 Stanley WC, Recchia FA, Lopaschuk GD. Myocardial substrate metabolism in the normal and failing heart. Physiol Rev 2005;85:1093-129.

38 Verma S, Rawat S, KL H. Empagliflozin increases cardiac energy production in diabetes. JACC Basic to Trans/ Sci 2018;3:575-87.

39 Goonasekera SA, Hammer K, Auger-Messier M, et al. Decreased cardiac L-type $\mathrm{Ca}^{2+}$ channel activity induces hypertrophy and heart failure in mice. J Clin Invest 2012;122:280-90.

40 Beuckelmann DJ, Näbauer M, Erdmann E. Intracellular calcium handling in isolated ventricular myocytes from patients with terminal heart failure. Circulation 1992;85:1046-55.

41 Banerjee SK, McGaffin KR, Pastor-Soler NM, et al. SGLT1 is a novel cardiac glucose transporter that is perturbed in disease states. Cardiovasc Res 2009;84:111-8.

42 Lee $\mathrm{T}-\mathrm{I}$, Chen Y-C, Lin Y-K, et al. Empagliflozin attenuates myocardial sodium and calcium dysregulation and reverses cardiac remodeling in streptozotocin-induced diabetic rats. Int J Mo/ Sci 2019;20. doi:10.3390/ijms20071680

43 Hammoudi $N$, Jeong D, Singh R, et al. Empagliflozin improves left ventricular diastolic dysfunction in a genetic model of type 2 diabetes. Cardiovasc Drugs Ther 2017:31:233-46.

44 Uthman L, Baartscheer A, Schumacher CA, et al. Direct cardiac actions of sodium glucose cotransporter 2 inhibitors target pathogenic mechanisms underlying heart failure in diabetic patients. Front Physiol 2018;9:1575.

45 Ng K-M, Lau Y-M, Dhandhania V, et al. Empagliflozin ameliorates high glucose induced-cardiac dysfuntion in human iPSC-derived cardiomyocytes. Sci Rep 2018:8:14872.
46 Baartscheer A, Schumacher CA, Wüst RCl, et al. Empagliflozin decreases myocardial cytoplasmic $\mathrm{Na}^{+}$through inhibition of the cardiac $\mathrm{Na}^{+} / \mathrm{H}^{+}$exchanger in rats and rabbits. Diabetologia 2017;60:568-73.

47 Uthman L, Baartscheer A, Bleijlevens B, et al. Class effects of SGLT2 inhibitors in mouse cardiomyocytes and hearts: inhibition of $\mathrm{Na}^{+} / \mathrm{H}^{+}$exchanger, lowering of cytosolic $\mathrm{Na}^{+}$and vasodilation. Diabetologia 2018;61:722-6.

48 Iborra-Egea O, Santiago-Vacas E, Yurista SR. Unraveling the molecular mechanism of action of empagliflozin in heart failure with reduced ejection fraction with or without diabetes. JACC Basic to Trans/ Sci 2019;4:831-40.

49 Maiuri MC, Zalckvar E, Kimchi A, et al. Self-eating and self-killing: crosstalk between autophagy and apoptosis. Nat Rev Mol Cell Biol 2007;8:741-52.

50 Santulli G. Cardioprotective effects of autophagy: eat your heart out, heart failure! $\mathrm{SCi}$ Trans/ Med 2018;10:eaau0462.

51 Luo G, Jian Z, Zhu Y. Sirt1 promotes autophagy and inhibits apoptosis to protect cardiomyocytes from hypoxic stress. Int J Mol Med 2020.

52 Zhou H, Wang S, Zhu P, et al. Empagliflozin rescues diabetic myocardial microvascular injury via AMPK-mediated inhibition of mitochondrial fission. Redox Biol 2018;15:335-46.

53 Hawley SA, Ford RJ, Smith BK, et al. The Na+/glucose cotransporter inhibitor canagliflozin activates AMPK by inhibiting mitochondrial function and increasing cellular AMP levels. Diabetes 2016;65:2784-94.

54 Esterline RL, Vaag A, Oscarsson J, et al. Mechanisms in endocrinology: SGLT2 inhibitors: clinical benefits by restoration of normal diurnal metabolism? Eur $\lrcorner$ Endocrinol 2018;178:R113-25.

55 Ghantous CM, Azrak Z, Hanache S, et al. Differential role of leptin and adiponectin in cardiovascular system. Int J Endocrinol 2015;2015:1-13.

56 lacobellis G, Ribaudo MC, Zappaterreno A, et al. Relation between epicardial adipose tissue and left ventricular mass. Am J Cardiol 2004;94:1084-7.

57 Schulze PC, Kratzsch J, Linke A, et al. Elevated serum levels of leptin and soluble leptin receptor in patients with advanced chronic heart failure. Eur J Heart Fail 2003:5:33-40.

58 Wu P, Wen W, Li J, et al. Systematic review and meta-analysis of randomized controlled trials on the effect of SGLT2 inhibitor on blood leptin and adiponectin level in patients with type 2 diabetes. Horm Metab Res 2019;51:487-94.

59 Scheen AJ. [EMPA-REG OUTCOME: Empagliflozin reduces mortality in patients with type 2 diabetes at high cardiovascular risk]. Rev Med Liege 2015;70:583-9 http:// www.ncbi.nlm.nih.gov/pubmed/26738271

60 Neal B, Perkovic V, Mahaffey KW, et al. Canagliflozin and cardiovascular and renal events in type 2 diabetes. N Engl J Med 2017;377:644-57.

61 Wiviott SD, Raz I, Bonaca MP, et al. Dapagliflozin and cardiovascular outcomes in type 2 diabetes. N Engl J Med 2019;380:347-57.

62 Cannon CP, Pratley R, Dagogo-Jack S, et al. Cardiovascular outcomes with ertugliflozin in type 2 diabetes. N Engl J Med 2020;383:1425-35.

63 McMurray JJV, Solomon SD, Inzucchi SE, et al. Dapagliflozin in patients with heart failure and reduced ejection fraction. N Eng/ J Med 2019;381:1995-2008.

64 Packer M, Anker SD, Butler J. Cardiovascular and renal outcomes with Empagliflozin in heart failure. N Engl J Med2020:NEJMoa2022190. 


\section{Correction: Sodium-glucose co-transporter 2 inhibitor therapy: mechanisms of action in heart failure}

Joshi SS, Singh T, Newby DE, et al. Sodium-glucose co-transporter 2 inhibitor therapy: mechanisms of action in heart failure. Heart 2021;107:1032-1038. doi:10.1136/heartjnl-2020-318060

This article has been corrected since it was first published. The provenance and peer review statement has been included.

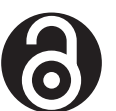

\section{OPEN ACCESS}

Open access This is an open access article distributed in accordance with the Creative Commons Attribution 4.0 Unported (CC BY 4.0) license, which permits others to copy, redistribute, remix, transform and build upon this work for any purpose, provided the original work is properly cited, a link to the licence is given, and indication of whether changes were made. See: https:// creativecommons.org/licenses/by/4.0/.

(C) Author(s) (or their employer(s)) 2021. Re-use permitted under CC BY. Published by BMJ.

Heart 2021;107:e15. doi:10.1136/heartjnl-2020-318060corr1

(D) Check for updates 